\title{
EL ARGUMENTO DE SUSTRACCIÓN PARA UNIVERSALES TRASCENDENTES
}

\author{
José Tomás Alvarado Marambio* \\ jose.tomas.alvarado@gmail.com
}

\begin{abstract}
This work presents the subtraction argument for transcendent universals, i.e., for universals that are not instantiated. The argument depends on two main premises: (i) the contingency of the different instantiations of a universal, and (ii) the ontological independence between these instantiations. By the first premise one can postulate metaphysically possible worlds where given instantiations of a universal are subtracted. One can consider, then, a possible world where only one object instantiates a universal. By the principle (ii) of independence, construed in a particular way, one can postulate in this point a possible world where no object instantiates the universal. It is contended that the independence thesis (ii) is too strong for the defender of immanent universal, who can construe it in a milder guise, compatible with the generic dependence of universals to having some or other instance.
\end{abstract}

Keywords transcendent universals; posible worlds; principle of independence; David M. Armstrong.

RESUMEN Este trabajo presenta el argumento de sustracción para defender los universales trascendentes, esto es, universales que no se encuentran instanciados. El argumento depende de dos premisas fundamentales: (i) la contingencia de las diferentes instanciaciones de un universal, y (ii) la independencia ontológica entre estas instanciaciones. Por

* Profesor asistente del Instituto de Filosofía de la Pontificia Universidad Católica de Chile. Artigo recebido em 17/12/2010 e aceito em 15/6/2011.

KRITERION, Belo Horizonte, nº 125, Jun./2012, p. 263-279. 
la primera premisa se pueden postular mundos metafísicamente posibles en donde las instanciaciones dadas de un universal son sustraidas. Se puede considerar, luego, un mundo posible en donde sólo un objeto instancia el universal. Por el principio (ii) de independencia, interpretado de un modo particular, se puede postular en este punto un mundo posible en donde ningún objeto instancia el universal. Se argumenta que la tesis de independencia (ii) es demasiado fuerte para el defensor de los universales inmanentes, quien puede entenderla de un modo más moderado, compatible con la dependencia genérica de los universales a tener una u otra instancia.

Palabras clave universales trascendentes; mundos posibles; principio de independencia; David M. Armstrong.

En los últimos decenios la postulación de universales ha ido ganando en aceptación. Parte importante de la motivación para que muchos filósofos se hayan sentido inclinados a postular la existencia de propiedades universales es el hecho de que los universales se han presentado como un complemento ontológico del realismo científico (cf. Armstrong, 1978a, 1978b; 1989a; 1997, 19-68, 113-138, 220-262). Los universales se han presentado, en efecto, como entidades que no se descubren por mera reflexión a priori acerca del contenido de nuestro pensamiento o de nuestro lenguaje, sino mediante investigación empírica. Estos universales, junto con explicar cómo es que algo uno puede darse en una multitud de instancias -el problema tradicional de los universalesson constituyentes de las leyes naturales, explicando cómo es que las leyes difieren de las meras regularidades (cf. Armstrong, 1983; Tooley, 1987, 37169; Dretske, 1977), y también explican por qué nuestras inducciones son habitualmente exitosas. Los universales que satisfacen todas estas funciones teóricas se han tomado habitualmente como universales inmanentes, esto es, como entidades que, aún cuando por su naturaleza pueden estar instanciadas en una pluralidad de ejemplificaciones, sólo existen si es que tienen instancias. Las propiedades postuladas son intrínsecas a los estados de cosas que constituyen.

Estas ventajas teóricas no parecen favorecer, sin embargo, a los universales trascendentes. Los universales trascendentes son entidades que, de acuerdo a su naturaleza, pueden estar instanciados en una pluralidad de ejemplificaciones, pero que no requieren estar instanciados para existir. Por este motivo, la inclinación hacia universales trascendentes ha sido mucho menor. En este trabajo se presentará y se discutirá un argumento que parece 
justificar la existencia de tales universales trascendentes, en contra de la corriente más dominante en metafísica de propiedades. Se ha denominado este argumento como "de sustracción" por analogía con otro argumento con semejanzas estructurales importantes para justificar la posibilidad de que no exista nada (cf. Baldwin, 1996). Aunque el veredicto será finalmente negativo acerca de la verosimilitud de este argumento de sustracción, servirá para poner de relieve las conexiones sistemáticas entre universales y particulares.

Para precisar un poco más el planteamiento de la cuestión, será conveniente especificar algunas tesis cruciales. La diferencia fundamental entre defensores de universales inmanentes y defensores de universales trascendentes reside en la actitud que se adopte ante el principio de instanciación:

\section{(1) $\square \forall \mathrm{X} \square \exists \mathrm{x}(\mathrm{x}$ es $\mathrm{X})$}

Aquí, la variable ' $\mathrm{X}$ ' tiene como rango universales y la variable ' $\mathrm{X}$ ' tiene como rango particulares. El principio de instanciación (1) establece que es necesario que todo universal posea tal naturaleza que se encuentre esencialmente instanciado en algún objeto u otro. El amigo de los universales trascendentes, naturalmente, negará el principio (1), esto es, aceptará que:

\section{(2) $\diamond \exists X \diamond \forall x \neg(x$ es $X)$}

Esto es, que es posible que exista un universal de tal naturaleza que pueda no estar instanciado por nada. El amigo de los universales trascendentes puede, adicionalmente a la aceptación de (2) admitir un principio más fuerte:

$$
\square \forall \mathrm{X} \square \exists \mathrm{Y}(\mathrm{Y}=\mathrm{X})
$$

Esto es, que es necesario que todo universal, en virtud de su naturaleza, exista de manera necesaria. (2) no implica (3), pues sería consistente que se admitiese que aunque algunos universales existen en algunos mundos posibles sin estar instanciados, algunos de esos universales no existen en todos los mundos posibles. Si se admite (3), por otro lado, y se asume que algunas de las instanciaciones de un universal son contingentes, se sigue (2). Es obvio, por otro lado, que el amigo de los universales inmanentes tendrá que rechazar (3). Los universales sólo existen en los mundos posibles en los que se encuentran instanciados. Bajo el mismo supuesto de que algunas de las instanciaciones de un universal son contingentes, se sigue que el universal en cuestión es una entidad contingente. La relación entre las posiciones en disputa podría verse como reflejada en un cuadrado de 
oposiciones en donde el platonismo fuerte y el aristotelismo (aceptación del principio de instanciación) son contrarios y se siguen las restantes relaciones de implicación usuales ${ }^{1}$ :

$\square \forall \mathrm{X} \square \exists \mathrm{x}(\mathrm{x}$ es $\mathrm{X})$

$\square \forall \mathrm{X} \square \exists \mathrm{Y}(\mathrm{Y}=\mathrm{X})$

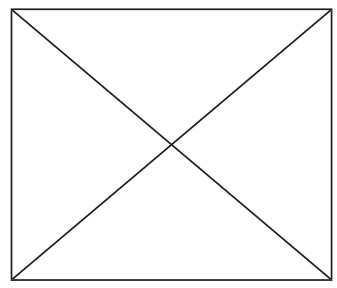

$\diamond \exists \mathrm{X} \diamond \forall \mathrm{Y}(\mathrm{Y} \neq \mathrm{X})$

$\diamond \exists X \diamond \forall \mathrm{x} \neg(\mathrm{x}$ es $\mathrm{X})$

La posición que acepta (3) se denominará 'platonismo fuerte' y la posición que sólo acepta (2) se denominará 'platonismo débil'. La posición que acepta (1) se denominará 'aristotelismo'. Para los propósitos de este trabajo basta considerar un platonismo débil, tal como se podrá apreciar, pero el argumento puede luego extenderse para el platonismo fuerte con relativa facilidad.

\section{El argumento de sustracción}

Un universal se instancia en particulares. Respecto del objeto particular, el hecho de instanciar ciertos universales es algo necesario o contingente. Esto es, hay ciertas propiedades esenciales que un objeto instancia en todo mundo posible en el que exista y hay otras propiedades que el objeto instancia en algunos mundos posibles en los que existe y no en otros. Algo semejante sucede cuando se considera la cuestión desde la perspectiva del universal que está siendo instanciado. Para un universal U puede resultar necesario o

1 Aquí [ $\square \forall X \square \exists x(x$ es $X)]$ es contradictoria de $[\diamond \exists X \backslash \forall \forall \neg(x$ es $X)$ ] y [ $\square \forall X \square \exists Y(Y=X)]$ es contradictoria de $[\diamond \exists X \diamond \forall Y(Y \neq X)] .[\nabla \exists X \diamond \forall Y(Y \neq X)]$ es subalterna de $[\square \forall X \square \exists x(x$ es $X)]$ y $[\diamond \exists X \diamond \forall x \neg(x$ es $X)]$ es subalterna de $[\square \forall X \square \exists Y(Y=X)]$. En el caso de las contrarias, $[\square \forall X \square \exists x(x$ es $X)]$ y $[\square \forall X \square \exists Y(Y=X)]$, la verdad de una de ellas implica la falsedad de la otra, pero la falsedad de una no implica la verdad de la otra. Por su parte, en las sub-contrarias, $[\diamond \exists X \diamond \forall Y(Y \neq X)]$ y $[\diamond \exists X \diamond \forall x \neg(X$ es $X)]$, la falsedad de una de ellas implica la verdad de la otra, pero la verdad de una no implica la falsedad de la otra. El platonismo débil puede tomarse de dos modos, de acuerdo a este esquema: (i) como la posición que acepta $[\diamond \exists X \diamond \forall X \neg(X$ es $X)]$, pero es neutral respecto del estatus de $[\square \forall \mathrm{X} \square \exists \mathrm{Y}(\mathrm{Y}=\mathrm{X})]$, o (ii) como la posición que junto con aceptar $[\diamond \exists X \diamond \forall \mathrm{X}$ $\neg(X$ es $X)$ ] acepta también $[\diamond \exists X \diamond \forall Y(Y \neq X)]$, esto es, como la posición que aceptando que no todos los universales deban encontrarse instanciados para existir, niega que todos los universales sean entidades necesarias. 
contingente el encontrarse instanciado en un objeto determinado. Si el objeto en cuestión es contingente, esto es, si el objeto en cuestión existe en ciertos mundos posibles, pero no en otros, entonces aunque el universal sea esencial al objeto, no será esencial al universal encontrarse instanciado por tal objeto, pues en los mundos posibles en los que el objeto no exista, el universal podrá todavía existir instanciado en otros objetos. Con mayor razón sucede esto si es que es contingente para un particular instanciar un universal U. En general, por lo tanto, las instanciaciones son contingentes para un universal $\mathrm{U}$, con la excepción del caso en que se trate de una propiedad esencial de un objeto necesario. Sólo en este caso el universal U no podrá existir sino instanciado en ese objeto, pues el objeto existe en todos los mundos posibles y en todos los mundos posibles en que existe debe instanciar U. No hay mundos posibles, entonces, en los que exista U y no esté instanciado por ese objeto. ¿Qué particulares satisfacen la condición de ser necesarios? Hay buenas razones para pensar que Dios, si existe, ha de ser necesario. Las entidades matemáticas han sido concebidas también usualmente como entidades necesarias. Estos casos serán, sin embargo, dejados a un lado, pues no serán relevantes para el argumento que se va a presentar.

Sea el universal $\mathrm{U}$ y los objetos $a_{1}, a_{2}, \ldots, a_{\mathrm{n}}$. Sea un mundo posible $\mathrm{w}_{1}$ en donde el universal $U$ está instanciado por cada uno de esos objetos $a_{1}, a_{2}, \ldots$, $a_{\mathrm{n}}$. Esto es, es verdadero en $\mathrm{w}_{1}$ que:

(4) $\left(a_{1}\right.$ es $\left.\mathrm{U}\right) \wedge\left(a_{2}\right.$ es $\left.\mathrm{U}\right) \wedge \ldots \wedge\left(a_{\mathrm{n}}\right.$ es $\left.\mathrm{U}\right)$

Pero, para U es contingente encontrarse instanciado por cada uno de $a_{1}$, $a_{2}, \ldots, a_{\mathrm{n}}$. Así, en $\mathrm{w}_{1}$ es también verdadero que:

(5) $\diamond \neg\left(a_{1}\right.$ es $\left.\mathrm{U}\right) \wedge \diamond \neg\left(a_{2}\right.$ es $\left.\mathrm{U}\right) \wedge \ldots \wedge \diamond \neg\left(a_{\mathrm{n}}\right.$ es $\left.\mathrm{U}\right)$

Se va a asumir en este argumento una lógica de tipo S5 para las relaciones de accesibilidad entre mundos posibles. Hay que suponer que la instanciación o no instanciación de $\mathrm{U}$ en cualquiera de los objetos $a_{1}, a_{2}, \ldots, a_{\mathrm{n}}$ no tiene ninguna implicación respecto de la instanciación de $U$ en cualquier otro objeto. Las instanciaciones de $\mathrm{U}$ en $\operatorname{los}$ objetos $a_{1}, a_{2}, \ldots, a_{\mathrm{n}}$ son independientes entre sí. Esto puede tomarse como:

(6) $\neg \square\left(\neg\left(a_{1}\right.\right.$ es $\left.\mathrm{U}\right) \rightarrow \exists \mathrm{x}(\mathrm{x}$ es $\left.\mathrm{U})\right) \wedge \neg \square\left(\neg\left(a_{2}\right.\right.$ es $\left.\mathrm{U}\right) \rightarrow \exists \mathrm{x}(\mathrm{x}$ es $\left.\mathrm{U})\right) \wedge \ldots$ $\wedge \neg \square\left(\neg\left(a_{\mathrm{n}}\right.\right.$ es $\left.\mathrm{U}\right) \rightarrow \exists \mathrm{x}(\mathrm{x}$ es $\left.\mathrm{U})\right)$ 
Pues bien, dado lo anterior, se puede pensar en un mundo posible $\mathrm{w}_{2}$ en que $\mathrm{U}$ no instancia uno de los objetos $a_{1}, a_{2}, \ldots, a_{\mathrm{n}}$. Así, en $\mathrm{w}_{2}$ sucede que:

(7) $\left(a_{1}\right.$ es $\left.\mathrm{U}\right) \wedge\left(a_{2}\right.$ es $\left.\mathrm{U}\right) \wedge \ldots \wedge\left(a_{\mathrm{n}-1}\right.$ es $\left.\mathrm{U}\right) \wedge \neg\left(a_{\mathrm{n}}\right.$ es $\left.\mathrm{U}\right)$

El procedimiento puede reiterarse $n$ veces hasta llegar a un mundo posible $\mathrm{w}_{\mathrm{n}}$ en que $\mathrm{U}$ sólo está instanciado por $a_{1}$ y nada más. En este mundo $\mathrm{w}_{\mathrm{n}}$ sigue siendo verdad (por S5), sin embargo, que:

(8) $\diamond \neg\left(a_{1}\right.$ es $\left.\mathrm{U}\right)$

Luego, hay un mundo posible $\mathrm{w}_{\mathrm{n}+1}$ en donde $\mathrm{U}$ no está instanciado por $a_{1}$. ¿Es necesario, sin embargo, que en este mundo $\mathrm{w}_{\mathrm{n}+1} \mathrm{U}$ esté instanciado en algún otro objeto? No. En efecto, de acuerdo a (6):

(9) $\neg \square\left[\neg\left(a_{1}\right.\right.$ es $\left.\mathrm{U}\right) \rightarrow \exists \mathrm{x}(\mathrm{x}$ es $\left.\mathrm{U})\right]$

Esto es, el hecho de no estar $\mathrm{U}$ instanciado por $a_{1}$ no implica nada respecto de la instanciación de U en otro objeto. (9) es equivalente a:

$$
\diamond\left[\neg\left(a_{1} \text { es } \mathrm{U}\right) \wedge \forall \mathrm{x} \neg(\mathrm{x} \text { es } \mathrm{U})\right]
$$

El mundo posible $\mathrm{w}_{\mathrm{n}+1}$ puede tomarse como un mundo posible en el que ni $a_{1}$ ni ningún otro objeto instancia $\mathrm{U}$. Por transitividad de las relaciones de accesibilidad, si $\mathrm{w}_{\mathrm{n}+1}$ es accesible respecto de $\mathrm{w}_{\mathrm{n}} \mathrm{y} \mathrm{w}_{\mathrm{n}}$ es accesible -por una cadena de pasos tal como los indicados- respecto de $\mathrm{w}_{1}$, entonces $\mathrm{w}_{\mathrm{n}+1}$ es accesible respecto de $\mathrm{w}_{1}$. Es posible, entonces, que el universal $U$ no esté instanciado, de lo que se sigue (2), la contradictoria del principio de instanciación (1).

El argumento de sustracción se sigue de las tres premisas (4), (5) y (6). La premisa (4) es trivial. Es simplemente suponer que un universal puede estar instanciado por varios objetos. Las premisas fundamentales son la (5) que establece la contingencia de las instanciaciones de un universal y la (6) que establece la independencia de las instanciaciones de un universal entre sí. Estas dos premisas parecen, por sí mismas, perfectamente razonables y algo cercano a ellas ha sido aceptado por los amigos de los universales inmanentes. Por ejemplo, Armstrong acepta expresamente que los estados de cosas que están constituidos por un universal y un particular que lo instancia son independientes entre sí (cf. Armstrong, 1997, 139-147). Esta 
independencia es lo que funda, por lo demás, su concepción combinatoria de la modalidad metafísica (cf. Armstrong, 1989b, 37-53, 77-86). Acepta también implícitamente la contingencia de las instanciaciones, pues los universales, aún cuando -en su teoría- deban encontrarse instanciados por algo para existir, no es necesario que estén instanciados por algún objeto específico.

El argumento de sustracción, entonces, sería un argumento para justificar que un universal podría no tener instancias. Esto es, es un argumento para el platonismo débil. No es difícil, sin embargo, argumentar a favor del platonismo fuerte si se acepta el platonismo débil. En efecto, suponer que hay universales no instanciados, pero suponer también que no todos los universales son entes necesarios es una posición metafísica mucho menos parsimoniosa que sostener simplemente que los universales son entidades necesarias y que, por lo tanto, no importa si están o no instanciados para existir. Suponiendo que un universal puede existir en un mundo posible sin estar instanciado, ¿por qué este universal no existe en otros mundos posibles? Debería entregarse una explicación razonable acerca de qué hace que exista o no exista en un mundo posible, si es que esa existencia contingente no depende de sus instanciaciones. Faltando esa explicación es más razonable suponer que si los universales son independientes de sus instanciaciones, entonces existen de manera invariante en todos los mundos posibles.

\section{Independencia y petición de principio}

Se podría alegar que el argumento de sustracción indicado incurre en petición de principio, pues la 'independencia' entre las instanciaciones -tal como se formula en (6)- parece ser, por sí misma, todo lo que necesita el amigo de los universales trascendentes. Tal como se ha indicado, en efecto, sostener para un objeto $a_{\mathrm{i}}$ cualquiera que su instanciación de $\mathrm{U}$ es 'independiente' de las restantes, según la formulación preferida, es ya sostener que:

(11) $\diamond\left[\neg\left(a_{\mathrm{i}}\right.\right.$ es $\left.\mathrm{U}\right) \wedge \forall \mathrm{x} \neg(\mathrm{x}$ es $\left.\mathrm{U})\right]$

De acuerdo al esquema $[\diamond(\alpha \wedge \beta) \rightarrow(\diamond \alpha \wedge \diamond \beta)]$, de (11) se sigue que:

(12) $\diamond \forall x \neg(x$ es $U)$

Pero (12) es simplemente sostener que es posible que U no sea instanciado por nada. Es, por lo tanto, justamente la conclusión que se desea obtener en virtud del argumento. Las restantes premisas parecen estar de más para esta 
conclusión. Esto hace dudar de que realmente el amigo de los universales inmanentes deba aceptar la formulación de la 'independencia' entre las instanciaciones tal como aparece en (6).

Pero cualquier conjunto de premisas del que se siga una conclusión $\mathrm{p}$ puede ser visto como una petición de principio contra quien no quiera aceptar p. Lo que consigue un buen argumento deductivo es hacer patente que la aceptación de ciertas premisas obliga a aceptar la conclusión. Lo que se ha hecho aquí es mostrar que todo aquel que acepte que las instanciaciones de un universal son contingentes y que son independientes entre sí, del modo indicado en (6), debe aceptar que hay universales no instanciados. El defensor de los universales inmanentes debe objetar las premisas, o bien objetar el paso de las premisas a la conclusión, pero no es objeción el simple hecho de que -en efecto- la conclusión se sigue de las premisas.

Si existen pruritos sobre la premisa (6), sin embargo, existe una forma de presentar la tesis de independencia de una manera más neutral y en la que no se siga de ella directamente la conclusión deseada. En vez de postular que el hecho de que un objeto $a_{\mathrm{i}}$ no instancie $\mathrm{U}$ no implica estrictamente que algo instancie U, se puede cualificar el consecuente de la implicación estricta negada del siguiente modo:

$$
\neg \square\left[\neg\left(a_{\mathrm{i}} \text { es } \mathrm{U}\right) \rightarrow \exists \mathrm{x}\left(\left(\mathrm{x} \neq a_{\mathrm{i}}\right) \wedge\left(a_{\mathrm{i}} \text { es } \mathrm{U}\right)\right)\right]
$$

Esto es, el hecho de que $a_{\mathrm{i}}$ no instancie $\mathrm{U}$ no implica estrictamente que algo diferente de $a_{\mathrm{i}}$ instancie U. De (13) se sigue que:

$$
\text { (14) } \diamond\left[\neg\left(a_{\mathrm{n}} \text { es } \mathrm{U}\right) \wedge \forall \mathrm{x}\left(\left(\mathrm{x} \neq a_{\mathrm{i}}\right) \rightarrow \neg(\mathrm{x} \text { es } \mathrm{U})\right)\right]
$$

Aplicando nuevamente el esquema $[\diamond(\alpha \wedge \beta) \rightarrow(\diamond \alpha \wedge \diamond \beta)]$ sobre (14) sólo arroja la tesis inocua:

\section{(15) $\diamond \forall \mathrm{x}\left[\left(\mathrm{x} \neq a_{\mathrm{i}}\right) \rightarrow \neg(\mathrm{x}\right.$ es $\left.\mathrm{U})\right]$}

Esta tesis (15), a diferencia de (12) no sostiene que U no está instanciado en nada, sino sólo que es posible que nada diferente de $a_{\mathrm{i}}$ instancie U. Esta modificación de la tesis de independencia debería ser igualmente aceptable y de ella se sigue, del mismo modo, la conclusión deseada del argumento de sustracción. En el mundo posible $\mathrm{w}_{\mathrm{n}}$ sólo el objeto $a_{1}$ instancia $\mathrm{U}$ y en ese mundo es posible que $a_{1}$ no instancie $U$ y que nada diferente de $a_{1}$ instancie $U$. Sea ese mundo $\mathrm{w}_{\mathrm{n}+1}$ tal como se explicó más arriba. 


\section{Formas alternativas de independencia}

Es conveniente aquí hacer una exploración más amplia de las cuestiones de dependencia ontológica implicadas. El amigo del aristotelismo buscará, naturalmente, una formulación de la independencia entre las instanciaciones que no tenga los costos metafísicos indicados y que, en particular, sea compatible con el hecho de que los universales deberían existir instanciados en algún objeto u otro. De acuerdo a las distinciones efectuadas por Lowe $(1998,136-153)^{2}$, existen varias formas en que puede ser comprendida la noción de dependencia ontológica. Una primera formulación sería:

(16) (x depende para su existencia de $y)={ }_{\text {def }} \square((x$ existe $) \rightarrow($ y existe $))$

Si se quiere fortalecer la dependencia ontológica para hacerla antisimétrica, se puede modificar la definición (16) del siguiente modo:

(17) (x depende fuertemente para su existencia de $y)={ }_{\text {def }}[\square((x$ existe $) \rightarrow$ $($ y existe $)) \wedge \neg \square(($ y existe $) \wedge($ x existe $))]$

Un tercer tipo de dependencia ontológica que puede ser aducida aquí es la "dependencia genérica", que puede ser definida del siguiente modo:

(18) (x depende genéricamente para su existencia de objetos del tipo F) $={ }_{\text {def }} \square(($ x existe $) \rightarrow \exists y$ Fy $)$

De acuerdo a la noción de dependencia que se tenga en vista será la noción de independencia que resulte, pues, en general, se puede definir que:

(19) (x es independiente para su existencia de $y)=_{\text {def }} \neg$ (x es dependiente para su existencia de $\mathrm{y})$

El problema central que surge aquí es que lo que ha sido negado en la premisa (6) de independencia es la dependencia genérica de un universal a tener instancias, esto es, lo que se ha negado es el hecho de que la no instanciación de $\mathrm{U}$ en el objeto $a_{\mathrm{i}}$ sea dependiente genéricamente en la instanciación de $\mathrm{U}$ en algún objeto u otro. Lo que debería sostener el defensor de los universales

2 Cf. también, Fine (1994-5). Fine refina la noción de dependencia ontológica de manera que la entidad $x$ sólo pueda decirse dependiente ontológicamente de la entidad y si es que, en virtud de la identidad de $\mathrm{x}$, si existe $\mathrm{x}$ debe existir también y. Esta cualificación no es relevante para lo que se discute aquí. 
inmanentes es la independencia de las instanciaciones particulares entre sí, junto con la dependencia del universal a tener genéricamente alguna instancia. Para cada uno de los objetos del dominio $a_{1,} a_{2}, \ldots, a_{\mathrm{n}}$ deberían haber tesis como la siguiente:

$(20) \neg \square\left(\neg\left(a_{\mathrm{i}}\right.\right.$ es $\left.\mathrm{U}\right) \rightarrow\left(a_{1}\right.$ es $\left.\left.\mathrm{U}\right)\right) \wedge \neg \square\left(\neg\left(a_{\mathrm{i}}\right.\right.$ es $\left.\mathrm{U}\right) \rightarrow\left(a_{2}\right.$ es $\left.\left.\mathrm{U}\right)\right) \wedge \ldots \wedge$ $\neg \square\left(\neg\left(a_{\mathrm{i}}\right.\right.$ es $\left.\mathrm{U}\right) \rightarrow\left(a_{\mathrm{n}}\right.$ es $\left.\left.\mathrm{U}\right)\right)$

Pero:

(21) $\square\left(\neg\left(a_{\mathrm{i}}\right.\right.$ es $\left.\mathrm{U}\right) \rightarrow \exists \mathrm{x}(\mathrm{x}$ es $\left.\mathrm{U})\right)$

La proposición (20) que establece la independencia de la no instanciación de $\mathrm{U}$ en $a_{\mathrm{i}}$ es independiente de la instanciación de $\mathrm{U}$ en todos los restantes objetos, por supuesto, no implica la negación de (21). En efecto, de (20) se sigue: es $\mathrm{U}) \wedge \neg\left(a_{\mathrm{n}}\right.$ es $\left.\left.\mathrm{U}\right)\right)$

(22) $\diamond\left(\neg\left(a_{\mathrm{i}}\right.\right.$ es $\left.\mathrm{U}\right) \wedge \neg\left(a_{1}\right.$ es $\left.\left.\mathrm{U}\right)\right) \wedge \diamond\left(\neg\left(a_{\mathrm{i}}\right.\right.$ es $\left.\mathrm{U}\right) \wedge \neg\left(a_{2}\right.$ es $\left.\left.\mathrm{U}\right)\right) \wedge \ldots \wedge \diamond\left(\neg\left(a_{\mathrm{i}}\right.\right.$

Por aplicación reiterada del esquema $[\diamond(\alpha \wedge \beta) \rightarrow(\diamond \alpha \wedge \diamond \beta)] \mathrm{y}$ simplificación se sigue de (22) que:

$$
\diamond \neg\left(a_{1} \text { es } \mathrm{U}\right) \wedge \diamond \neg\left(a_{2} \text { es } \mathrm{U}\right) \wedge \ldots \wedge \diamond \neg\left(a_{\mathrm{n}} \text { es } \mathrm{U}\right)
$$

Pero, obviamente, esto no implica que:

$$
\diamond\left[\neg\left(a_{1} \text { es } \mathrm{U}\right) \wedge \neg\left(a_{2} \text { es } \mathrm{U}\right) \wedge \ldots \wedge \neg\left(a_{\mathrm{n}} \text { es } \mathrm{U}\right)\right]
$$

Lo indicado en (24) es la posibilidad contemplada, sin embargo, cuando se postula la independencia genérica a tener alguna instancia, tal como ha aparecido en (6). Resulta, por lo tanto, que el amigo de los universales inmanentes tiene a su disposición una tesis mucho más débil de independencia entre las instanciaciones de un universal que respeta, sin embargo, la dependencia genérica del universal a tener instancias. El argumento de sustracción parece estar neutralizado. 


\section{Una objeción y su réplica}

El defensor de los universales trascendentes podría en este punto aducir que la dependencia genérica enunciada en la proposición (21) es muy poco verosímil como tesis ontológica. El argumento de sustracción tendría la virtud de poner de relieve cómo el amigo de los universales inmanentes se ve obligado a aceptar tal tesis, para evitar el funcionamiento del argumento. En la medida en que la tesis en cuestión sea efectivamente un costo muy alto que pagar ganará también en plausibilidad la aceptación de universales trascendentes. La proposición (21) establece, en efecto, que la no-instanciación de U en un objeto cualquiera $a_{\mathrm{i}}$ hace necesario que $U$ sea instanciado en algún otro objeto. ¿No es extraño postular que una no-instanciación haga necesario un estado de cosas? Muchas teorías modales siguen una inspiración básicamente humeana en que cualquier cosa puede darse junto a cualquier otra, o separada de cualquier otra, en la medida en que se trate de existentes diferentes. Esto es lo que postula David Lewis para conformar el espacio modal de sus mundos posibles (cf. Lewis, 1986a, 86-92). También una idea semejante tiene una función rectora en la metafísica modal combinatoria de David Armstrong y otros (cf. Armstrong, 1989b, 37-53; 1997, 139-174; Skyrms, 1981). Pues bien, si cualquier cosa podría darse junto con cualquier otra o separada de cualquier otra, ¿por qué el hecho de que un objeto $a_{\mathrm{i}}$ no instancie $\mathrm{U}$ hace necesaria la instanciación de U? ¿Qué hay de tan especial en tal no-instanciación que impone una restricción tan severa a las combinaciones permisibles de objetos y propiedades? No parece claro que existan respuestas satisfactorias a estas preguntas para el amigo de los universales inmanentes.

Hay otras concepciones modales, sin embargo, en donde el espacio modal no viene dado por meras recombinaciones de un repertorio de entidades independientes entre sí. En estas teorías lo metafísicamente posible y lo metafísicamente necesario son constituidos por disposiciones o poderes causales de un carácter primitivo (cf. Molnar, 2003, 200-223; Mumford, 2004, 160-181; Borghini y Williams, 2008; Alvarado, 2009). Algo es posible porque hay poderes causales que podrían haber hecho ese estado de cosas efectivo. Pues bien, ¿qué tipo de poder causal posee la no-instanciación de $U$ en $a_{\mathrm{i}}$ tal que hace necesario que $U$ se instancie en otro objeto? Los hechos negativos no poseen poderes causales ${ }^{3}$, por lo que es muy difícil hacer comprensible cómo es que podría ser verdadero un principio de dependencia genérico tal

3 En teorías anti-realistas de la causalidad los hechos negativos o eventos negativos pueden entrar en relaciones causales con otros hechos o eventos (cf., por ejemplo, Lewis, 1986b, 189-193; 2004) . Estas teorías anti-realistas, sin embargo, no pueden entrar en una concepción modal fundada en poderes 
como (21). En uno u otro caso, por lo tanto, el principio (21) parece poco verosímil. Si la postulación de universales inmanentes conduce a aceptar este principio inverosímil, entonces hay motivos para rechazar que los universales sólo existen si es que poseen instancias.

Una objeción de este estilo puede ser neutralizada también por el amigo de los universales inmanentes mediante un par de analogías. Hay otras estructuras ontológicas en las que también se presenta una dependencia genérica junto con la independencia particular entre varios ítems entre sí. En estos casos, la aplicación de algo análogo al argumento de sustracción llevaría, sin embargo, a resultados desastrosos. Considérese, en primer lugar, la relación que existe entre un objeto material y la materia de que está compuesto. En las ontologías tradicionales la composición no es identidad (cf. Lowe, 1998, 28209; Wiggins, 2001, 21-138). Un objeto material es numéricamente diferente del material de que está compuesto. El mismo pedazo de materia $m$ puede constituir el objeto $a_{1}$ en el instante de tiempo $t_{1}$ y luego constituir el objeto $a_{2}\left(a_{1} \neq a_{2}\right)$ en el instante de tiempo $t_{2}$. El mismo pedazo de materia $m$ puede constituir el objeto $a_{1}$ en el mundo posible $\mathrm{w}_{1} \mathrm{y}$ constituir el objeto $a_{2}\left(a_{1} \neq\right.$ $a_{2}$ ) en el mundo posible $\mathrm{w}_{2}$. Un objeto podría estar compuesto de un material diferente de aquel de que está de hecho compuesto, al menos para instantes de tiempo posteriores al instante de origen de ese objeto. Considérese un organismo vivo como, por ejemplo, el gato Micifuz. Micifuz está compuesto por una pluralidad de moléculas orgánicas, pero podría estar compuesto por moléculas orgánicas numéricamente diferentes de aquellas que lo conforman. Para cada una de las moléculas orgánicas que componen a Micifuz es posible que no estén componiendo el cuerpo de Micifuz. A su vez, el hecho de una molécula orgánica específica no esté constituyendo el cuerpo de Micifuz es independiente del hecho de que otras moléculas orgánicas estén componiendo el cuerpo de Micifuz. Si se toman estas dos tesis del mismo modo que se han tomado las premisas (5) y (6) del argumento de sustracción resulta una conclusión absurda. Sea que:

(25) $\left(m_{1}\right.$ compone el cuerpo de Micifuz $) \wedge\left(m_{2}\right.$ compone el cuerpo de Micifuz $) \wedge \ldots \wedge\left(m_{\mathrm{n}}\right.$ compone el cuerpo de Micifuz $)$

(26) $\diamond \neg\left(m_{1}\right.$ compone el cuerpo de Micifuz $) \wedge \diamond \neg\left(m_{2}\right.$ compone el cuerpo de Micifuz $) \wedge \ldots \wedge \diamond \neg\left(m_{\mathrm{n}}\right.$ compone el cuerpo de Micifuz $)$ 
La independencia entre estas moléculas componiendo el organismo de Micifuz de manera análoga a (6) sería:

(27) $\neg \square\left(\neg\left(m_{1}\right.\right.$ compone el cuerpo de Micifuz) $\rightarrow \exists x$ (x compone el cuerpo de Micifuz $) \wedge \neg \square\left(\neg\left(m_{2}\right.\right.$ compone el cuerpo de Micifuz $) \rightarrow \exists x$ (x compone el cuerpo de Micifuz $) \wedge \ldots \wedge \neg \square\left(\neg\left(m_{\mathrm{n}}\right.\right.$ compone el cuerpo de Micifuz $) \rightarrow \exists \mathrm{x}$ (x compone el cuerpo de Micifuz))

Aunque en este caso no parece tener sentido ir sustrayendo una a una las moléculas del cuerpo de Micifuz sin sustituirlas por otras, de (27) se sigue, por lo que respecta a $m_{1}$, que:

(28) $\diamond\left(\neg\left(m_{1}\right.\right.$ compone el cuerpo de Micifuz) $\wedge \neg \exists x$ (x compone el cuerpo de Micifuz))

Y luego:

(29) $\diamond \neg \exists x$ (x compone el cuerpo de Micifuz)

Pero es ridículo pensar que Micifuz podría no estar compuesto por ninguna molécula de orgánica. El argumento conduciría a la postulación de un gato inmaterial o algo semejante.

Otro caso en el que se presenta una estructura ontológica semejante es en el caso de las propiedades determinadas y las propiedades determinables (cf. Armstrong, 1978b, 111-113). Una propiedad determinada es, por ejemplo, la propiedad de tener exactamente una masa de $n$ gramos, por oposición a la propiedad determinable de tener una u otra masa. Si un objeto tiene una propiedad determinable, entonces debe tener una propiedad determinada (si algo tiene masa, entonces tiene una masa determinada). Si un objeto tiene una propiedad determinada, entonces debe tener una propiedad determinable correlativa (si algo tiene una masa determinada, entonces tiene masa). Si un objeto tiene una propiedad determinada, entonces no tendrá ninguna otra propiedad determinada bajo el mismo determinable (si algo tiene una propiedad de exactamente $n$ gramos, entonces no tendrá ninguna masa de $n+i$, ó $n-i$ para un $i$ real cualquiera). Sea, por ejemplo, una superficie $s$ con un color determinado $\mathrm{C}_{1}$. Sean $\mathrm{C}_{1}, \mathrm{C}_{2}, \ldots, \mathrm{C}_{\mathrm{n}}$ todos los colores determinados. Es contingente para la superficie $s$ poseer el color $\mathrm{C}_{1}$, pues podría tener un color diferente. El hecho de que $s$ no tenga el color $\mathrm{C}_{1}$ es independiente del hecho de que tenga otro color determinado. Si se interpretan estas tesis de un modo 
análogo a como se hace en (5) y (6) en el argumento de sustracción, resulta, sin embargo, un desastre. Sea que:

(30) $\left[(s\right.$ tiene color $) \wedge\left(s\right.$ es $\left.\left.\mathrm{C}_{1}\right)\right]$

(31) $\diamond\left[(s\right.$ tiene color $) \wedge \neg\left(s\right.$ es $\left.\left.\mathrm{C}_{1}\right)\right]$

Supóngase ahora que se introduce una tesis de independencia entre los colores determinados de $s$ análoga a como esto se hace en (6) para el argumento de sustracción:

(32) $\neg \square\left[\left((s\right.\right.$ tiene color $) \wedge \neg\left(s\right.$ es $\left.\left.\mathrm{C}_{1}\right)\right) \rightarrow \exists \mathrm{X}((\mathrm{X}$ es un color $) \wedge(s$ es $\left.\mathrm{X}))\right]$

Nuevamente, se seguiría aquí de (32) que:

(33) $\diamond\left[(s\right.$ tiene color $) \wedge \neg\left(s\right.$ es $\left.\mathrm{C}_{1}\right) \wedge \forall \mathrm{X}((\mathrm{X}$ es un color $) \rightarrow \neg(s$ es $\left.\mathrm{X}))\right]$

Y luego:

(34) $\diamond[(s$ tiene color $) \wedge \forall \mathrm{X}((\mathrm{X}$ es un color $) \rightarrow \neg(s$ es $\mathrm{X}))]$

Esto es, sería posible que $s$, teniendo color, no tuviese ningún color determinado. Es característico en estos casos que se introducen principios de independencia genéricos, tal como el introducido en (6). En estos dos casos sucede, también, que el resultado desastroso puede ser evitado si es que se debilita la tesis de independencia. Para el gato Micifuz se podría postular por cada una de las moléculas orgánicas que lo componen que:

(35) $\neg \square\left(\neg\left(m_{\mathrm{i}}\right.\right.$ compone el cuerpo de Micifuz $) \rightarrow\left(m_{1}\right.$ compone el cuerpo de Micifuz $) \wedge \neg \square\left(\neg\left(m_{\mathrm{i}}\right.\right.$ compone el cuerpo de Micifuz $) \rightarrow\left(m_{2}\right.$ compone el cuerpo de Micifuz $)) \wedge \ldots \wedge \neg \square\left(\neg\left(m_{\mathrm{i}}\right.\right.$ compone el cuerpo de Micifuz $) \rightarrow\left(m_{\mathrm{n}}\right.$ compone el cuerpo de Micifuz))

Junto con sostener que hay una dependencia genérica en estar compuesto por una molécula orgánica u otra. Del mismo modo, para la superficie $s$ se puede postular que:

(36) $\neg \square\left(\left((s\right.\right.$ tiene color $) \wedge \neg\left(s\right.$ es $\left.\left.\mathrm{C}_{1}\right)\right) \rightarrow\left(s\right.$ es $\left.\left.\mathrm{C}_{2}\right)\right) \wedge \ldots \wedge \neg \square(((s$ tiene color $) \wedge \neg\left(s\right.$ es $\left.\left.\mathrm{C}_{1}\right)\right) \rightarrow\left(s\right.$ es $\left.\left.\mathrm{C}_{\mathrm{n}}\right)\right)$ 
Junto con sostener que hay una dependencia genérica a poseer algún color determinado u otro. Entonces, pareciera que hay un problema más profundo con el argumento de sustracción. Si éstas son maniobras aceptables para rescatar la inteligibilidad de la composición material o de la relación entre propiedades determinadas y determinables, entonces no se ve porqué no sería también aceptable para hacer inteligible a los universales inmanentes.

Estas analogías también pueden ayudar a comprender por qué la dependencia genérica de un universal inmanente a poseer instancias no debe tomarse como una tesis ontológicamente extravagante. Un organismo vivo es una entidad que consiste en cierta estructuración de una pluralidad de materiales. Poseer un color es poseer un color determinado u otro. El organismo depende genéricamente en moléculas orgánicas porque, aunque no sea necesario que esté constituida por moléculas orgánicas determinadas, debe estar constituida por algunas, si es que ese organismo va a existir. La propiedad determinable de tener un color depende genéricamente de los colores determinados porque, aunque no sea necesario tener un color determinado, algo que tiene un color debe tener algún color determinado, si es que va a darse un color. De un modo análogo, el defensor de los universales inmanentes puede alegar que existir para un universal inmanente es estar instanciado en un objeto u otro, aunque no sea necesario que el universal esté instanciado en un objeto específico y por ello el universal depende genéricamente en la posesión de instanciaciones. Nada debe resultar ontológicamente extravagante de esta situación, si es que tampoco son extravagantes las estructuras ontológicas de la composición material y la relación entre propiedades determinables y propiedades determinadas.

\section{Conclusiones}

Se ha presentado el argumento de sustracción para justificar la existencia de universales trascendentes. El argumento depende, fundamentalmente, de dos premisas: la contingencia de las instanciaciones especificas de un universal y la independencia entre estas instanciaciones, entendida de un modo específico. Si se excluye el caso de las propiedades esenciales de entidades necesarias, las instanciaciones de un universal parecen contingentes y la primera premisa resulta, por lo tanto, perfectamente plausible. En cuanto a la segunda premisa de independencia, también parece plausible por sí misma. Los defensores del aristotelismo han aceptado estas dos premisas ordinariamente y han fundado teorías importantes en ellas, como Armstrong lo ha hecho con la teoría combinatoria de la modalidad que descansa, precisamente, en que 
un universal podría estar instanciado en otros objetos diferentes de aquellos en que está instanciado y en que las instanciaciones o no-instanciaciones que tenga un universal no hacen necesarias otras instanciaciones en otros objetos. Los supuestos de contingencia de las instanciaciones e independencia de las instanciaciones, entendido de este modo, conducen, conjuntamente, a admitir la posibilidad de que un universal exista sin instanciaciones. Como ninguna instanciación de un universal dado hace necesaria ninguna otra del mismo universal, entonces se pueden ir 'sustrayendo' tales instanciaciones hasta postular un mundo metafísicamente posible sin instanciaciones.

Se han considerado algunas objeciones contra esta argumentación. La premisa de independencia puede cualificarse si es que pareciere que implica demasiado directa y obviamente la conclusión deseada. Esta cualificación conduce, sin embargo, al mismo resultado. Se ha mostrado, sin embargo, que hay una forma de formular la tesis de independencia de un modo que la hace coherente con el principio de instanciación. En esta formulación alternativa, se preserva la independencia entre las instanciaciones específicas de un universal, manteniendo, sin embargo, la dependencia genérica del universal a poseer instancias.

Se considera, luego, si la dependencia ontológica genérica propuesta entre la no-instanciación de un universal y otras instanciaciones pudiese ser vista como extraña y repugnante por igual a filósofos de inclinaciones humeanas y no humeanas en metafísica modal. Se argumenta, sin embargo, que nada hay de especialmente extraño en la dependencia genérica apuntada. Estructuras semejantes pueden ser encontradas en la composición material de un objeto y en la relación existente entre una propiedad determinada y la propiedad determinable correlativa. En estos casos la introducción de una tesis de independencia semejante a la introducida para el argumento de sustracción conduciría a resultados desastrosos. Si es razonable en esos casos imponer una dependencia genérica, también lo es en el caso de los universales inmanentes. El argumento de sustracción no parece, por lo tanto, convincente 4 . 


\section{Referencias bibliográficas}

ALVARADO, José Tomás (2009), “Una teoría causal de la modalidad” Ideas y valores 140, 173-196.

ARMSTRONG, David M. (1978a), Universals and Scientific Realism, Volume I, Nominalism and Realism, Cambridge: Cambridge University Press.

ARMSTRONG, David M. (1978b), Universals and Scientific Realism, Volume II, A Theory of Universals, Cambridge: Cambridge University Press.

ARMSTRONG, David M. (1983), What is a Law of Nature?, Cambridge: Cambridge University Press.

ARMSTRONG, David M. (1989a), Universals. An Opinionated Introduction, Boulder: Westview.

ARMSTRONG, David M. (1989b), A Combinatorial Theory of Possibility, Cambridge: Cambridge University Press.

ARMSTRONG, David M. (1997), A World of States of Affairs, Cambridge: Cambridge University Press.

BALDWIN, Thomas (1996), "There Might Be Nothing” Analysis 56, 231-238.

BORGHINI, Andrea y WILLIAMS, Neil (2008), "A Dispositional Theory of Possibility" Dialectica 62, 21-41.

COLLINS, John, Ned Hall y L. A. Paul (2004) (eds.), Causation and Counterfactuals, Cambridge, Mass.: MIT Press.

DRETSKE, Fred (1977), "Laws of Nature" Philosophy of Science 44, 248-268.

FINE, Kit (1994-5), "Ontological Dependence" Proceedings of the Aristotelian Society 95, 269-290.

LEWIS, David (1986a), On the Plurality of Worlds, Oxford: Blackwell.

LEWIS, David (1986b), Postcripts a "Causation" en Philosophical Papers, Volume II, Oxford: Oxford University Press, 172-213.

LEWIS, David (2004), "Void and Object" en Collins, Hall y Paul (2004), 277-290.

LOWE, Jonathan (1998), The Possibility of Metaphysics. Substance, Identity, and Time, Oxford: Clarendon Press.

MOLNAR, George (2003), Powers. A Study in Metaphysics, Oxford: Oxford University Press.

MUMFORD, Stephen (2004), Laws in Nature, London: Routledge.

SKYRMS, Brian (1981), “Tractarian Nominalism” Philosophical Studies 40, 199-206. TOOLEY, Michael (1987), Causation. A Realist Approach, Oxford: Clarendon Press. WIGGINS, David (2001), Sameness and Substance Renewed, Cambridge: Cambridge University Press. 